[Article]

\title{
葡萄糖苷酶抑制剂作用机理的分子动力学模拟和自由能计算
}

\author{
罗 芳 ${ }^{1}$ 高 剑 ${ }^{1}$ 成元华 ${ }^{1,2}$ 崔 巍 $^{1}$ 计明娟 ${ }^{1, *}$ \\ ('中国科学院研究生院化学与化学工程学院, 北京 100049; \\ 2清华大学化学系, 教育部有机光电分子工程重点实验室, 北京 100084)
}

\begin{abstract}
摘要：含有锍离子的葡萄糖苷酶抑制剂如 kotalanol (SK)和它除去磺酸基团后的衍生物(DSK), 是潜在的毒副 作用较小的治疗 II 型糖尿病的候选药物. $\alpha$-葡萄糖苷酶抑制活性实验显示, DSK 活性比 SK略高, 而将二者环上 的 S原子替换成 NH 后(分别称为 DSN 和 SN), DSN 的活性要比 SN 高 1500 倍左右. 本文用分子动力学模拟, 结 合自由能计算和自由能分解的方法对上述四个抑制剂的作用机理进行了研究. 研究结果表明活性的巨大差异 是由 $\mathrm{NH}$ 基团取代效应和磺酸基团立体效应共同作用的结果, 由于 $\mathrm{N}-\mathrm{C}$ 键长比 $\mathrm{S}-\mathrm{C}$ 键长短, $\mathrm{NH}$ 基团取代导 致烷基链的翻转, 同时, 磺酸基团限制了链的翻转, 因此改变了抑制剂的结合模式. 计算结果与实验基本一致. 本文的研究结果有助于进一步理解含锍离子的葡萄糖苷酶抑制剂的结合机理, 并为设计更有潜力的葡萄糖苷 酶抑制剂提供了有价值的信息.
\end{abstract}

关键词：锍离子；葡萄糖苷酶抑制剂；分子动力学模拟；自由能计算； 自由能分解 中图分类号: 0641

\section{Interaction Mechanisms of Inhibitors of Glucoamylase by Molecular Dynamics Simulations and Free Energy Calculations}

\author{
LUO Fang ${ }^{1} \quad$ GAO Jian ${ }^{1} \quad$ CHENG Yuan-Hua ${ }^{1,2} \quad$ CUI Wei ${ }^{1} \quad J I M i n g-J u a n^{1, *}$ \\ ('College of Chemistry and Chemical Engineering, Graduate University of Chinese Academy of Sciences, Beijing 100049, \\ P. R. China; ${ }^{2}$ Key Laboratory of Organic Optoelectronics and Molecular Engineering of Ministry of Education, \\ Department of Chemistry, Tsinghua University, Beijing 100084, P. R. China)
}

\begin{abstract}
Sulfonium ion glucosidase inhibitors such as kotalanol (SK) and de-O-sulfonated kotalanol (DSK) are potential drug candidates for the treatment of type II diabetes, with no serious toxicity or side effects. Experimental binding assays against glucosidase show that the activity of DSK is slightly higher than that of SK, while the activity of the nitrogen analogue of de-O-sulfonated kotalanol (DSN) is $\sim 1500$-fold higher than that of the nitrogen analog of kotalanol (SN). Here, the binding mechanisms of four representative inhibitors of glucoamylase, SK, DSK, and their two nitrogen analogues, were explored in an integrated modeling study combining molecular dynamics (MD) simulations, binding free energy calculations, and binding free energy decomposition analysis. Our simulations highlight the significant impact of the combination of nitrogen substitution and sulfate anion group. Nitrogen substitution in the five-membered ring leads to the overturning of the polyhydroxylated chain, originating from the shorter bond length of $\mathrm{N}-\mathrm{C}$ compared with $\mathrm{S}-\mathrm{C}$, while the sulfate anion group restrains the freedom of the polyhydroxylated chain. These cumulative effects are able to significantly change the binding conformation of the inhibitor and substantially impair interactions between the inhibitor and glucosidase. The structural insights obtained in this study are expected to be valuable for increased understanding of the binding
\end{abstract}

Received: May 7, 2012; Revised: July 6, 2012; Published on Web: July 6, 2012.

"Corresponding author. Email: jmj@gucas.ac.cn; Tel: +86-10-88256326.

The project was supported by the National Natural Science Foundation of China (21173264), National Science and Technology Major Special Project of China (2009ZX09501-011), and Foundation of Knowledge Innovative Engineering of Chinese Academy of Sciences (ZNWH-2011-011).

国家自然科学基金(21173264), 科技部重大专项(2009ZX09501-011)和中国科学院知识创新工程基金(ZNWH-2011-011)资助项目

(C) Editorial office of Acta Physico-Chimica Sinica 
mechanism of sulfonium ion glucosidase inhibitors and future design of more potent glucosidase inhibitors.

Key Words: Sulfonium ion; Glucoamylase inhibitor; Molecular dynamic simulation; Free energy calculation; Free energy decomposition

\section{Introduction}

In human, four enzymes, i.e., salivary, pancreatic $\alpha$-amylases, maltase-glucoamylase, and sucrase-isomaltase, are involved in the complete digestion of starch into glucose. Maltase-glucoamylase (MGAM) belongs to the family GH31 of glycoside hydrolases and is a key intestinal human glucosidase responsible for the digestion of terminal starch products left after $\alpha$-amylase action. ${ }^{1}$ Anchored to the small-intestinal brush-border epithelial cells, it contains two catalytic subunits: an N-terminal subunit (ntMGAM) that is proximal to the membrane-bound end and a C-terminal luminal subunit (ctMGAM). The catalytic subunits have various but overlapping substrates, including maltose, isomaltose, sucrose, and small linear and branched oligosaccharides. ${ }^{2,3}$ Due to their involvement in the breakdown of dietary sugars and starches, the inhibition of MGAM by $\alpha$-glucosidase inhibitors can be a means of controlling postprandial blood glucose levels for individuals with type II diabetes. ${ }^{4}$ This class of inhibitors can also be used for acquired immune deficiency syndrome (AIDS) treatment. ${ }^{5}$ Acarbose and miglitol are currently used to control blood glucose levels by inhibiting amylases and glucosidase. They keep blood sugar levels within a safe range by slowing the rate at which the intestines absorb sugar (glucose) from food. Previous studies of the interaction between $\alpha$-glucosidase and acarbose, miglitol and glycosidase indicated that the carbohydrate mimics containing nitrogen are protonated in the active site of glucosidase and act as glucosidase inhibitors due to their similarity in the shape and charge to the presumed transition state for enzymatic glycoside hydrolysis.

The $\alpha$-glucosidase inhibitors with sulfonium ions were first isolated from Salacia reticulate, a plant that is widely used in traditional Ayurvedic medicine for treating type II diabetes in Sri Lanka and South India.? The Salacia extract was shown to be effective for the treatment of type II diabetes with less toxicity and side effects compared to the existing commercial glucosidase inhibitors that may cause liver dysfunction. ${ }^{8}$ Six active compounds have been isolated from the extract, including salaprinol, ${ }^{9}$ salacinol, ${ }^{10}$ ponkoranol, ${ }^{9}$ kotalanol (SK), ${ }^{11}$ de- $O$-sulfonated kotalanol (DSK), ${ }^{12}$ and de- $O$-sulfonated salacinol ${ }^{13}$ All of these compounds share a common structural motif: a zwitterionic sulfonium sulfate structure comprising a 1,4-anhydro4-thio-D-arabinitol and a polyhydroxylated acyclic chain. Ponkoranol and kotalanol differ in the length of the polyhydroxylated chain where ponkoranol has a six-carbon chain whereas kotalanol has a seven-carbon chain. The permanent positive charge of the sulfur atom is supposed to bind in the same way as acarbose and miglitol in the active sites of glucosidases. ${ }^{14}$
Sim et $a l^{15}$ recently reported the X-ray crystallographic structures of ntMGAM in complex with three $\alpha$-glucosidase inhibitors derived from the natural extracts of Salacia reticulate, including salacinol, SK, and DSK and they found that among these three inhibitors DSK is the most potent one with a inhibition constant $\left(K_{\mathrm{i}}\right)$ value of $30 \mathrm{nmol} \cdot \mathrm{L}^{-1}$. The synthesis and biological evaluation of the nitrogen analogues of SK (SN) and DSK (DSN) ${ }^{16}$ show that the activity of DSK is slightly higher than that of SK, while the activity of DSN is $\sim 1500$-fold higher than that of SN. Until now there have been few theoretical studies on the effect of heteroatom substitution regarding this class of inhibitors, and an atomic level understanding of the substitution effect has not been achieved and there still remains some debates regarding several aspects of the interaction mechanism. ${ }^{17}$ In the current work, the interactions between ntMGAM and four inhibitors (SK, DSK SN, and DSN) were investigated by molecular dynamics (MD) simulations, molecular mechanics/generalized born surface area (MM/GBSA) free energy calculations, and MM/GBSA free energy decomposition analysis. The structural and energetic insights obtained here are helpful for designing more potent inhibitors of glucosidase.

\section{Materials and methods}

\subsection{Preparation of the inhibitors}

The structures and inhibitory activities ${ }^{15,16}$ of the studied glucosidase inhibitors are summarized in Table 1.

The starting structures for the MD simulations of ntMGAM in complex with SK and DSK were retrieved from the protein data bank (PDB) (PDB entries: 3L4V and 3L4U). ${ }^{15}$ The structures of the other two inhibitors were built by replacing the ring sulfur atom in SK or DSK with a nitrogen atom (SN or DSN). The missing hydrogen atoms of the inhibitors and the proteins were added using the tleap program in AMBER 11. ${ }^{18}$ The inhibitors were minimized using the HF/6-31G* optimization in Gaussian 03 program, ${ }^{19}$ and the partial charges were obtained by fitting the electrostatic potentials derived by Gaussian program via the RESP fitting technique ${ }^{20}$ in AMBER11. The partial charges and the force field parameters for the inhibitors were generated by the antechamber program in AMBER11.

\subsection{Molecular dynamics simulations}

The general AMBER force field (GAFF) ${ }^{21}$ was chosen for the inhibitors and the AMBER03 force field ${ }^{22}$ for the protein. All the systems were immersed in a truncated octahedral box with TIP3P water molecules, ${ }^{23}$ and the crystallographic waters from the X-ray experiments were retained. $\mathrm{Na}^{+}$ions were added to maintain electrostatic neutrality. Energy minimizations 
Table 1 Structures and inhibitory activities of the studied molecules

Name

and MD simulations were carried out by using the sander module in AMBER11. The whole systems were minimized in three stages to remove bad contacts. Firstly, the water molecules were minimized by restraining the protein; secondly, water and the side chains of the protein were minimized by restraining the backbone of the protein, and each stage was performed by using the steepest descent minimization of 2500 steps followed by a conjugate gradient minimization of 2500 steps; thirdly, the entire system was minimized without any restriction by 10000 steps starting with the steepest descent minimization followed by the conjugate gradient minimization after 5000 cycles. The system was then heated gradually from 0 to $310 \mathrm{~K}$ in the NVT ensemble and equilibrated at $310 \mathrm{~K}$ for another $60 \mathrm{ps}$, and then $14 \mathrm{~ns}$ MD simulations were performed at a constant temperature of $310 \mathrm{~K}$ and a constant pressure of $10^{5} \mathrm{~Pa}$. During the sampling process, the coordinates were saved every 1 ps and the conformations generated from the last 2 ns simulations were used for further binding free energy calculations and decomposition analysis. Particle mesh ewald (PME) was employed to deal with the long-range electrostatic interactions in a periodic boundary condition. ${ }^{24} \mathrm{~A}$ cutoff equal to $1 \mathrm{~nm}$ was used for short-range electrostatics and van der Waals interactions and a continuum model correction term was added up to the van der Waals energies. Temperature was regulated using the weak-coupling algorithm ${ }^{25}$ with the time constant of $0.5 \mathrm{ps}$. The SHAKE method was used to constrain hydrogen atoms and the time step was set to $2 \mathrm{fs}^{26}$

\subsection{MM/GBSA free energy calculations and free energy decomposition analysis}

The obtained stable MD trajectory for each complex was used to estimate the binding free energy $\left(\Delta G_{\text {bind }}\right)$ using the $\mathrm{MM} /$ GBSA technique implemented in AMBER11. ${ }^{27-37}$ According to the previous studies, MM/GBSA showed better performance to rank the binding affinities for systems without metals than molecular mechanics/poisson boltzmann surface area (MM/PBSA) ${ }^{38}$ In MM/GBSA, the binding free energy $\left(\Delta G_{\text {bind }}\right)$ between a ligand $(\mathrm{L})$ and a receptor $(\mathrm{R})$ is calculated by the following equation:

$$
\begin{aligned}
\Delta G_{\text {bind }} & =G_{\text {complex }}-G_{\text {protein }}-G_{\text {ligand }} \\
& =\Delta H+\Delta G_{\text {solvation }}-T \Delta S \\
& \approx \Delta E_{\mathrm{MM}}+\Delta G_{\mathrm{GB}}+\Delta G_{\mathrm{SA}}-T \Delta S
\end{aligned}
$$

where $\Delta E_{\mathrm{MM}}$ accounts for the non-bonded interaction energy in the gas phase that encompasses the terms for electrostatic interaction energy $\left(\Delta E_{\text {ele }}\right)$, the van der Waals interaction energy $\left(\Delta E_{\mathrm{vdw}}\right)$, and the difference of internal energy $\left(\Delta E_{\text {inter }}\right)$. In this case the difference of internal energy is zero because the single MD trajectory was used to derive the energy components for ligand, receptor, and complex; $\Delta G_{\mathrm{GB}}$ and $\Delta G_{\mathrm{SA}}$ are the polar and non-polar components of the desolvation free energy, respectively; $-T \Delta S$ is the change of conformational entropy upon ligand binding, which was estimated by using normal-mode analysis. Considering the expensive computational cost, only the residues in the catalytic domain of protein were included in the calculations of entropy, and 50 snapshots taken at an interval of $40 \mathrm{ps}$ from the final $2 \mathrm{~ns}$ of the MD simulation were used to estimate the conformational entropy. The polar component of desolvation $\left(\Delta G_{\mathrm{GB}}\right)$ was calculated with a generalized Born model $(\mathrm{GB})^{39,40}$ of the AMBER suite. The non-polar component was determined by solvent accessible surface area (SASA) using the LCPO method: ${ }^{41} \Delta G_{\mathrm{SA}}=0.0072 \Delta \mathrm{SASA}$, where $\triangle \mathrm{SASA}=\mathrm{SASA}_{\text {complex }}-\left(\mathrm{SASA}_{\text {protein }}+\mathrm{SASA}_{\text {ligand }}\right)$ and the solvent accessible surface areas were calculated with a solventprobe radius of $0.14 \mathrm{~nm}$. For each system, free energy calculations were performed for 200 snapshots extracted from the last 2 ns MD trajectories.

The interactions between each residue in protein and each inhibitor were analyzed using the MM/GBSA free energy decomposition analysis applied in the mm_pbsa module in AMBER11. This analysis is essential as it facilitates us to discover the key residues which contribute more to the ligand binding with the residues which are responsible for the different binding affinities of the four inhibitors. The binding interaction between each residue and an inhibitor includes three terms: van der Waals contribution $\left(\Delta G_{\mathrm{vdw}}\right)$, electrostatic contribution $\left(\Delta G_{\text {ele }}\right)$, and desolvation contribution $\left(\Delta G_{\text {solvation }}\right)$.

$$
\begin{aligned}
\Delta G_{\text {residue-inhibitor }} & =\Delta G_{\text {vdw }}+\Delta G_{\text {ele }}+\Delta G_{\text {solvation }} \\
& =\Delta G_{\text {vdw }}+\Delta G_{\text {ele }}+\Delta G_{\mathrm{GB}}+\Delta G_{\mathrm{SA}}
\end{aligned}
$$

All energy components in Eq.(2) were calculated using the same snapshots as the free energy calculation. The polar contribution of desolvation energy $\left(\Delta G_{\mathrm{GB}}\right)$ was estimated by using GB models, and the parameters for GB calculations were developed by Onufriev et al. (igb=2). ${ }^{42}$ The exterior dielectric con- 
stant was set to 80 , and the solute dielectric constant value was set to $4 .^{38}$ The non-polar contribution of desolvation $\left(\Delta G_{\mathrm{SA}}\right)$ was determined by SASA using the ICOSA technique. ${ }^{43}$

\section{Results and discussion}

\subsection{Stability and flexibility of the complexes}

To explore the stability and dynamic properties of the four complexes, 14 ns MD simulations were performed. The rootmean-square-deviation (RMSD) values of the protein backbone atoms relative to the initial structures were calculated and shown in Fig.1. The plot indicates that the DSK complex achieves equilibrium at around $500 \mathrm{ps}$, and fluctuates around $0.10-0.15 \mathrm{~nm}$, while the DSN complex reaches equilibrium at around $4000 \mathrm{ps}$, and fluctuates around $0.11-0.17 \mathrm{~nm}$. The SK and SN complexes reach equilibrium at around 5000 ps. All of the four complexes achieve equilibrium after $5000 \mathrm{ps}$, and the MD trajectories taken from the last 2000 ps simulations were used for the following analysis.

\subsection{Validation of the binding modes predicted by the MD simulations}

To evaluate the prediction accuracy of the MD simulations, the predicted structures of the last snapshot from the MD simulations for the four complexes were superimposed to the crystal structures of the DSK and SK complexes (Fig.2). The binding pocket of ntMGAM is comprised of the -1 and +1 subsites. ${ }^{44}$ From Fig.2(A, C), we can see that the ring part of the inhibitors occupies the -1 subsite and polyhydroxylated chain
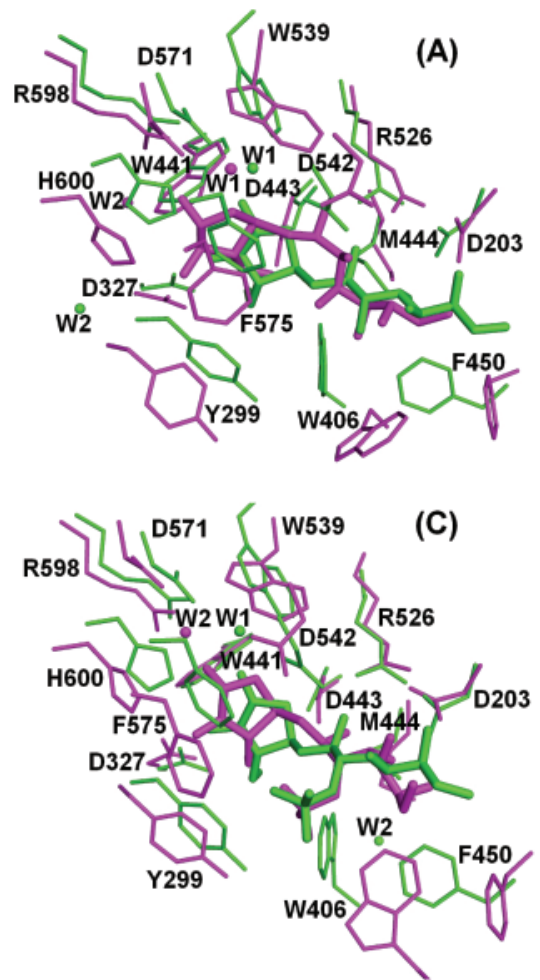

Fig.2 Comparison of the ntMGAM-inhibitor active site

(A) the MD and crystal structures of the DSK complex; (B) the MD structure of the DSN complex and the crystal structure of the DSK complex; (C) the MD and crystal structures of the SK complex; (D) the MD structure of the SN complex and the crystal structure of the SK complex. green: crystal structure, purple: MD structure; stick, line, and ball are used to represent bound inhibitors, residues, and conserved water molecules, respectively.

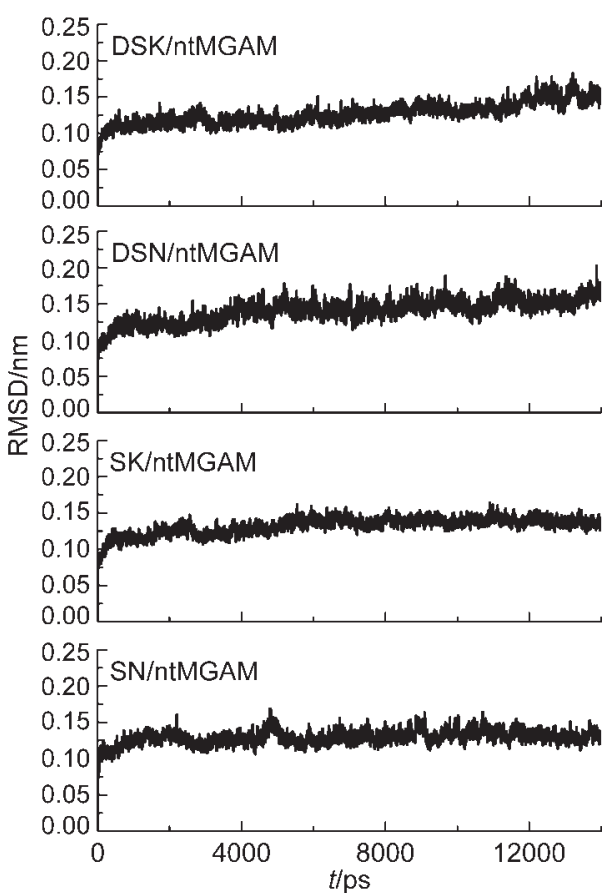

Fig.1 Time dependence of RMSD of the backbone atoms $\left(\mathrm{C}_{a}, \mathrm{~N}\right.$, and $\left.\mathrm{C}\right)$ with respect to the first snapshot of the inhibitor/ntMGAM complexes

part of them occupies the +1 subsite, which is in agreement with the experiment report. ${ }^{43}$ The MD structures of DSK and SK have a little difference from the crystal structures of the
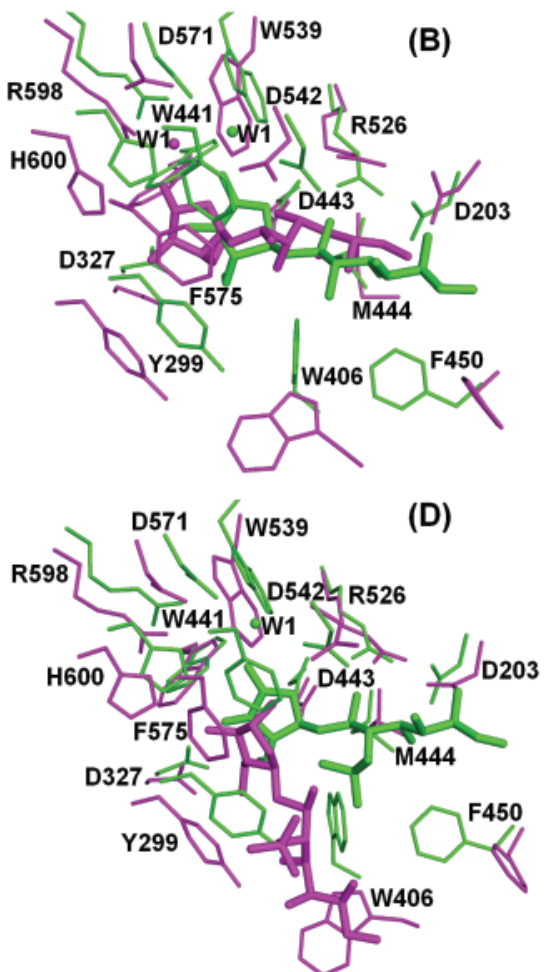
DSK and SK, we think that it is because of the moving of water molecules, which results in the moving of inhibitors. Compared to the orientations of $\mathrm{C}^{\prime} \mathrm{OH}$ in DSK and SK, which are very different in DSN and in SN and they turn up. Most interesting finding is that the predicted $\mathrm{SN}$ in the binding site from the MD simulations is substantially different from SK in the crystal structure. Quite different from the conformations of the other inhibitors in the binding site, SN does not form effective interactions with the -1 subsite. That is to say, the stable structure of SN predicted by the MD simulations is quite different from the initial structure modified from SK for the MD simulations.

\subsection{MM/GBSA binding free energy analysis}

The binding free energies were calculated for all the complexes using the MM/GBSA method and summarized in Table 2. It is encouraging that the predicted binding free energies ( $\Delta G_{\text {bind }}$ ) of the inhibitors are almost consistent with the experimental values except for the DSN complex. One possible reason for this inconsistency is that the difference of activity between DSK and DSN is so small. Moreover, DSK and DSN are positively charged, and the desolvation energies for the charged systems cannot be well predicted in many cases. ${ }^{38,45}$ The data in Table 2 demonstrate that the contribution of the non-polar part $\left(\Delta E_{\mathrm{vdw}}+\Delta G_{\mathrm{SA}}\right)$ is more favorable than that of the polar part $\left(\Delta E_{\mathrm{ele}}+\Delta G_{\mathrm{GB}}\right)$ because the electrostatic interaction is effectively neutralized by $\Delta G_{\mathrm{GB}}$. The van der Waals interaction $\left(-112.44 \mathrm{~kJ} \cdot \mathrm{mol}^{-1}\right)$ between SN and ntMGAM is more favorable than that between DSK and ntMGAM $\left(-93.72 \mathrm{~kJ} \cdot \mathrm{mol}^{-1}\right)$ and that between DSN and ntMGAM $\left(-107.37 \mathrm{~kJ} \cdot \mathrm{mol}^{-1}\right)$. Compared to those of SK and SN complexes, the polar contributions for DSK and DSN complexes are much stronger $\left(-18.88\right.$ and $-19.63 \mathrm{~kJ} \cdot \mathrm{mol}^{-1}$, respectively). It is not surprising because DSK and DSN are positively charged, and they tend to form stronger electrostatic interactions with the negatively charged residues in the binding sites than SK and SN. The electrostatic interactions for the SK and SN complexes are not strong enough to compensate the unfavorable desolvation contributions, hence leading to overall more unfavorable binding affinities than DSK and DSN. Compared with that for the SK complex $\left(5.48 \mathrm{~kJ} \cdot \mathrm{mol}^{-1}\right)$, the polar contribution $\left(\Delta E_{\text {ele }}+\Delta G_{\mathrm{GB}}\right)$ for the $\mathrm{SN}$ complex $\left(16.37 \mathrm{~kJ} \cdot \mathrm{mol}^{-1}\right)$ is more unfavorable. The difference of the polar contribution $\left(\Delta E_{\text {ele }}+\Delta G_{\mathrm{GB}}\right)$ between the four inhibitors is higher than that of the non-polar contribution $\left(\Delta E_{\mathrm{vdw}}+\Delta G_{\mathrm{SA}}\right)$, which maybe lead to the difference of their inhibitory activities.

\subsection{Structure-binding affinity relationship analysis}

From Fig. 2 we can see that the ring hydroxyl groups of the inhibitors form close contacts with the side chains of the residues D327, W441, F575, H600 and water molecules in the -1 subsite. The hydroxyl groups in the acyclic chains of the inhibitors can interact with the side chains of D203, R526, and D542 in the +1 subsite except for the SN complex. There are some hydrophobic amino acids in the active site of ntMGAM, such as W406, W441, W539, Y299, and F575, which can form van der Waals interactions with the inhibitors. Compare to the crystal structure, our MD simulation structures of inhibitors have a little difference due to the moving of water molecule, which indicates that the role of water molecule in the active site is important. By comparing the structures of the inhibitors and their binding modes three factors are found to be important for the difference of inhibitory activities: (a) role of the sulfate anion group, (b) effect of the nitrogen atom substitution, and (c) role of water in the active site.

In order to elucidate the important residues involved in protein-inhibitor interaction, the total binding free energy for each inhibitor was decomposed into residue-inhibitor pairs by using the MM/GBSA free energy decomposition analysis. . $^{34,43,46}$ The interaction patterns between the inhibitors and the residues of ntMGAM are shown in Figs.3-5.

\subsubsection{Role of the sulfate anion group}

In general, de- $O$-sulfonation leads to an increase of affinity compared to the parent sulfated compounds because removing the sulfate group enhances the freedom of the polyhydroxylated chain of DSK, thus making it be easier to interact with the residues in the active site. ${ }^{15}$

As can be seen from Fig. 3 and Fig.4, compared with the de- $O$-sulfonated compounds (DSN and DSK), the parent sulfated compounds (SN and SK) have stronger van der Waals interactions with Y299 and W406. The impact of the sulfate anion group on glycosidase inhibitory activity was inferred by Yuasa et $a l .^{47}$ by docking salacinol into the active site of glucoamylase. Their study illustrates that the sulfate ion can form strong non-covalent interaction with the arginine residues in the binding site. However, Sim et al. ${ }^{15}$ found that the sulfate group does not form any significant hydrogen bonding interactions with the ntMGAM active site but seems to be constrained by Y299, W406, and F575. Mohan et al. ${ }^{16}$ proposed that the positioning of the sulfate anion of $\mathrm{SN}$ in a hydrophobic pocket in the active site is more sterically compromised than that of SK.

According to Yuasa's opinion, ${ }^{47}$ the positively charged argi-

Table 2 Binding free energies and the individual energy terms predicted by MM/GBSA

\begin{tabular}{|c|c|c|c|c|c|c|c|c|c|}
\hline Name & $\Delta E_{\mathrm{ele}} /\left(\mathrm{kJ} \cdot \mathrm{mol}^{-1}\right)$ & $\Delta E_{\mathrm{vdv}}\left(\mathrm{kJ} \cdot \mathrm{mol}^{-1}\right)$ & $\Delta G_{\mathrm{SA}}\left(\mathrm{kJ} \cdot \mathrm{mol}^{-1}\right)$ & $\Delta G_{\mathrm{GB}} /\left(\mathrm{kJ} \cdot \mathrm{mol}^{-1}\right)$ & $\Delta G_{\text {polar }} /\left(\mathrm{kJ} \cdot \mathrm{mol}^{-1}\right)$ & $T \Delta S /\left(\mathrm{kJ} \cdot \mathrm{mol}^{-1}\right)$ & $\Delta G_{\text {bind }} /\left(\mathrm{kJ} \cdot \mathrm{mol}^{-1}\right)$ & $\Delta G_{\text {exp }} /\left(\mathrm{kJ} \cdot \mathrm{mol}^{-1}\right)$ & $K_{\mathrm{i}}\left(\mathrm{nmol} \cdot \mathrm{L}^{-1}\right)$ \\
\hline DSK & $-645.02 \pm 16.83$ & $-93.72 \pm 18.17$ & $-20.55 \pm 1.42$ & $626.14 \pm 14.40$ & $-18.88 \pm 5.27$ & $-90.59 \pm 29.76$ & -42.57 & -44.54 & $30^{15}$ \\
\hline DSN & $-638.95 \pm 11.93$ & $-107.37 \pm 18.25$ & $-18.75 \pm 1.25$ & $619.32 \pm 9.46$ & $-19.63 \pm 5.02$ & $-88.49 \pm 36.46$ & -57.26 & -42.70 & $61^{16}$ \\
\hline SK & $-156.64 \pm 13.10$ & $-111.47 \pm 21.01$ & $-23.48 \pm 0.84$ & $162.17 \pm 9.13$ & $5.48 \pm 6.53$ & $-108.38 \pm 32.69$ & -21.10 & -39.77 & $190^{15}$ \\
\hline $\mathrm{SN}$ & $-104.27 \pm 10.17$ & $-112.44 \pm 12.85$ & $-20.01 \pm 0.80$ & $120.64 \pm 8.20$ & $16.37 \pm 4.19$ & $-106.95 \pm 21.56$ & -9.13 & -23.94 & $90000^{16}$ \\
\hline
\end{tabular}

$\Delta E_{\text {ele }}$, electrostatic energy; $\Delta E_{\mathrm{vdw}}$, van der Waals energy; $\Delta G_{\mathrm{GB}}$, the polar desolvation energy; $\Delta G_{\mathrm{SA}}$, nonpolar desolvation energy; 

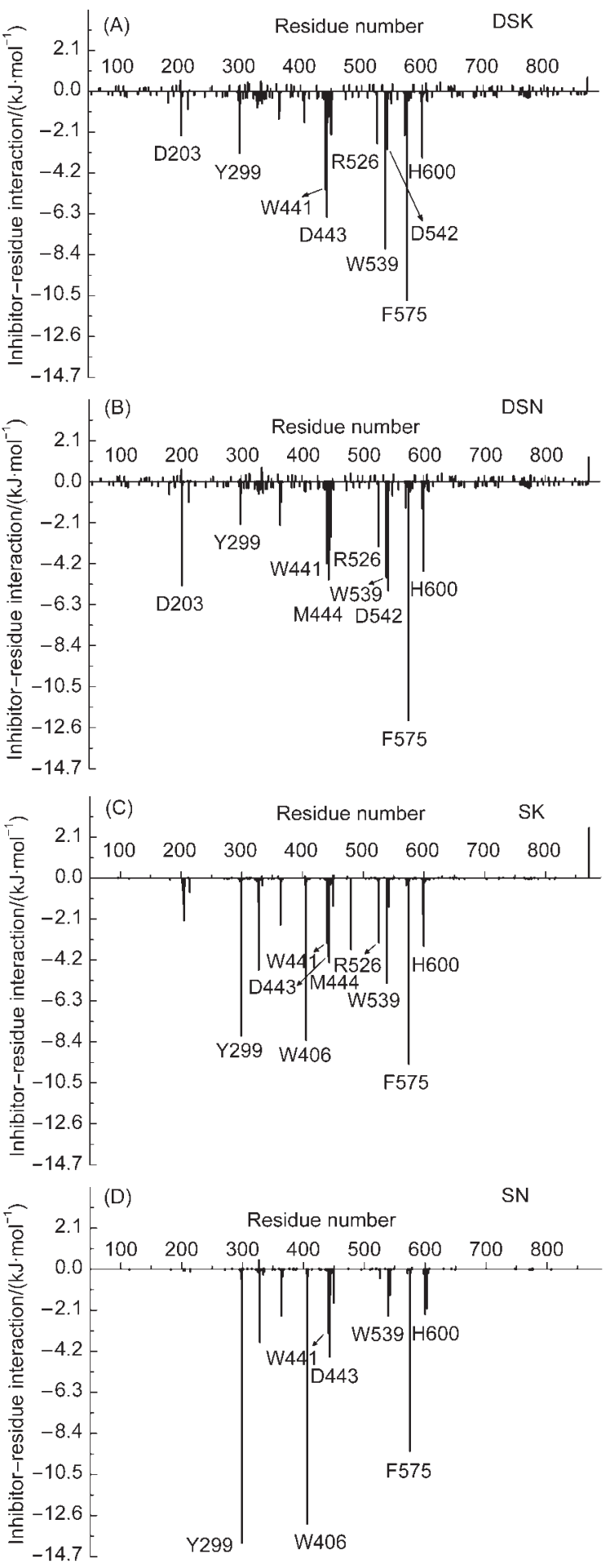

Fig.3 Inhibitor-residue interaction spectra for

(A) the DSK complex, (B) the DSN complex, (C) the SK complex, and (D) the SN complex

nine can form strong electrostatic interaction with the negative charge of the sulfate group. As shown in Fig.4 we cannot find favorable electrostatic interaction between any arginine in the active site and the inhibitor, and there are only unfavorable electrostatic interactions between the inhibitors and the residue R526, which is not consistent with the Yuasa's theory.
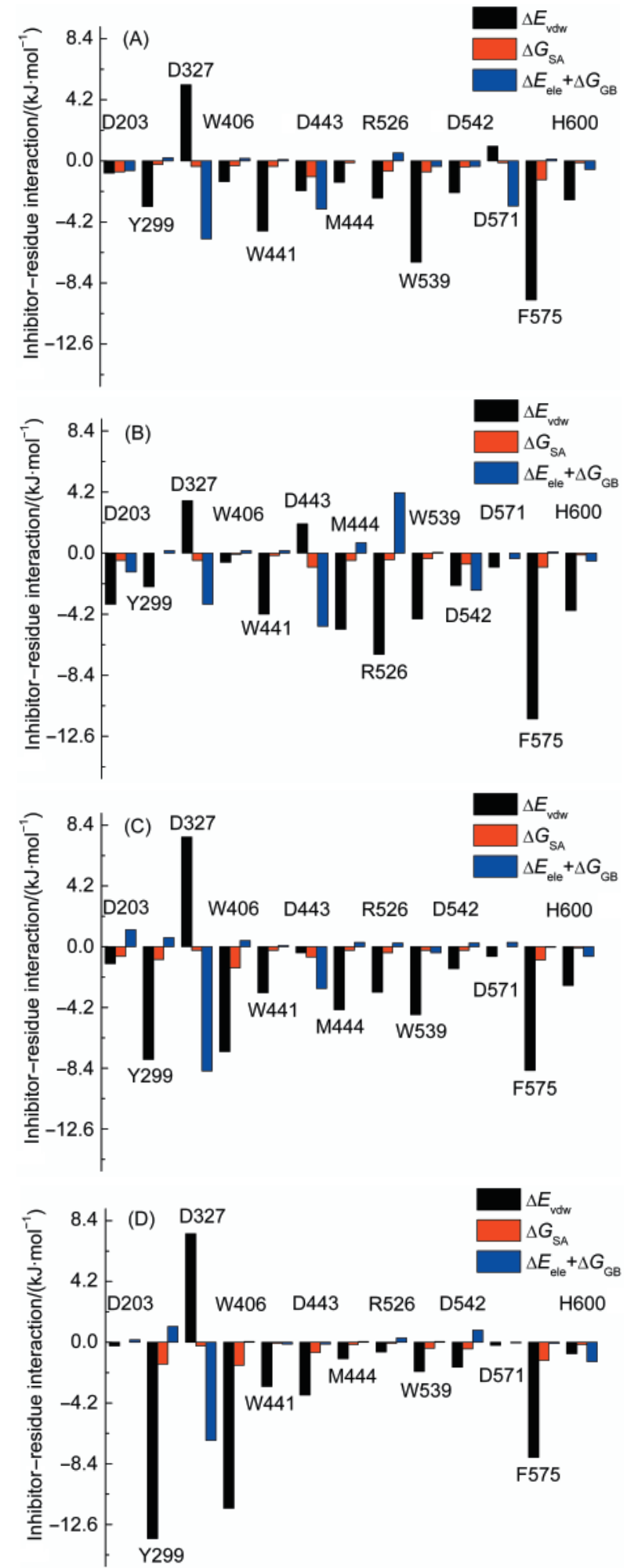

Fig.4 Inhibitor-residue interaction spectra of

individual energy terms for the residues in the active site for (A) the DSK complex, (B) the DSN complex, (C) the SK complex, and (D) the SN complex

In order to understand if the sulfate group can form stable hydrogen bonds with the residues in the active site, the hydrogen bond ratio was analyzed by the ptraj module in AMBER11. The 

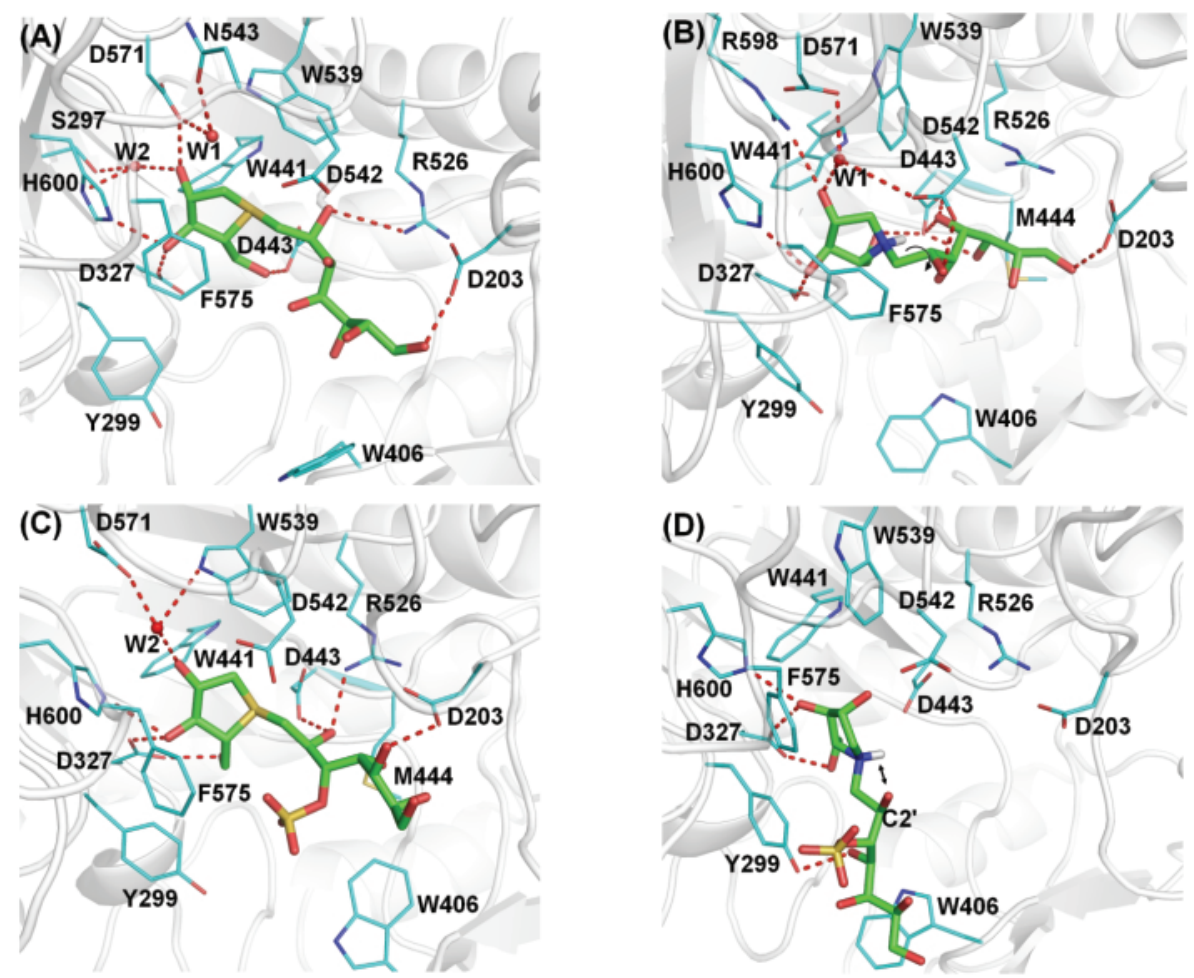

Fig.5 Structural representations for the studied inhibitors in complex with ntMGAM (A) the DSK complex, (B) the DSN complex, (C) the SK complex, (D) the SN complex

order number of $\mathrm{O}$ atom is shown in Fig.6. The important hydrogen bonding interactions in all the complexes are summarized in Table 3. A hydrogen bond is defined by a distance $(<0.35 \mathrm{~nm})$ and an orientation (the angle $\theta(\mathrm{A} \cdots \mathrm{H}-\mathrm{D})>$ $120.0^{\circ}$ ). Our analysis shows that stable hydrogen bonding interaction between the sulfate group and Y299 may exist in the SK complex, and it is between the $\mathrm{H}$ atom of hydroxyl in Y299 and the three equivalent $\mathrm{O}$ atoms connected by the sulfur atom of the sulfate anion. The hydrogen bond ratios between SK and Y299 are $26.65 \%, 22.45 \%$, and $16.35 \%$, respectively. Moreover, there is no hydrogen bonding interaction between the sulfate group and Y299 in the SN complex and Y299 can form a stable hydrogen bond with the $\mathrm{O} 4$ atom of $\mathrm{SN}$, and the corresponding hydrogen bond ratio is $98 \%$.

Due to the structural difference between $\mathrm{C}^{\prime} \mathrm{OH}$ in DSN and in SN, SN is closer to Y299 and W406 than DSN, and therefore, the van der Waals interaction between $\mathrm{SN}$ and the resi-
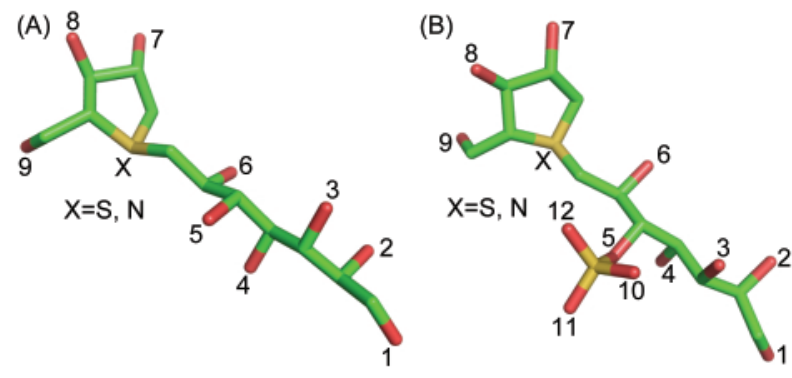

Fig.6 Order numbers of the oxygen atoms in (A) DSK or DSN and (B) SK or SN dues Y299 and W406 is stronger than that between SK and the residues Y299 and W406 (Fig.4). Meanwhile, the distances between SN and D542, R526, D443 are increased and the corresponding interactions are decreased. The difference of the inhibitory activities between DSK and SK suggests that the effect of the sulfate anion group on activity is not significant.

\subsubsection{Impact of nitrogen atom substitution}

Mohan $^{16}$ and Eskandari ${ }^{48}$ et al. studied the effect of the replacement of ring sulfur atom by nitrogen and selenium on inhibitory activity, but they did not elucidate the mechanism at atomic level. Compared to sulfur, the smaller radius of nitrogen accounts for its shorter bond length of $\mathrm{N}-\mathrm{C}(0.15 \mathrm{~nm})$ than that of $\mathrm{S}-\mathrm{C}(0.18 \mathrm{~nm})$, which leads to the repulse interaction between $\mathrm{C}^{\prime} \mathrm{OH}$ and the $\mathrm{H}$ atom of NH. Fig.5(D) shows that the polyhydroxylated chain of SN is close to the side chain of W406. It is possible that the repulse interaction between $\mathrm{C}^{\prime} \mathrm{OH}$ and $\mathrm{NH}$ leads to a trend of turning up for $\mathrm{C}^{\prime} \mathrm{OH}$, and due to the static effect between sulfate group and Y299, the turnover is inhibited. As shown in Fig.5, it is obvious that SK can form more hydrogen bonds with ntMGAM than SN. Fig.5 (B) shows that removing the sulfate group relieves the positional constraint imposed by the bulky hydrophobic residues surrounding the $\mathrm{C}^{\prime} \mathrm{OH}$ group, and then the whole polyhydroxylated chain can turn up freely in the DSN complex. We can also observe that the contribution of F575 for the DSN complex is stronger $\left(-12.22 \mathrm{~kJ} \cdot \mathrm{mol}^{-1}\right)$ than those for the other three complexes (Fig.3 and Fig.4), because the turnover of the $\mathrm{C}^{\prime} \mathrm{OH}$ group in polyhydroxylated chain of DSN enhances the interac- 
Table 3 Hydrogen bond analysis from the last 2 ns MD

\begin{tabular}{|c|c|c|c|c|}
\hline Name & Acceptor & Donor & Occupancy $/ \%$ & Distance $/ \mathrm{nm}$ \\
\hline \multirow[t]{11}{*}{ DSK complex } & W2@O & :S297@HG-:S297@OG & 98.65 & 0.2820 \\
\hline & D571@OD1 & :DSK@H5-:DSK@O7 & 98.25 & 0.2718 \\
\hline & D327@OD2 & :DSK@H9-:DSK@O8 & 91.40 & 0.2621 \\
\hline & DSK@06 & :R526@HH11-:R526@NH1 & 88.65 & 0.2947 \\
\hline & DSK@O8 & :H600@HE2-:H600@NE2 & 68.95 & 0.2894 \\
\hline & D443@OD2 & :DSK@H2-:DSK@O9 & 55.40 & 0.2756 \\
\hline & D571@OD1 & :W1@H1-:W1@O & 55.10 & 0.2750 \\
\hline & N543@OD1 & :W1@H2-:W1@O & 52.70 & 0.2818 \\
\hline & DSK@07 & :W2@H2-:W2@O & 43.95 & 0.2961 \\
\hline & H600@ND1 & :W2@H1-:W2@O & 30.30 & 0.3015 \\
\hline & D203@OD1 & :DSK@H1-:DSK@O1 & 25.50 & 0.2820 \\
\hline \multirow[t]{10}{*}{ DSN complex } & D327@OD2 & :DSN@H21-:DSN@O8 & 100.00 & 0.2592 \\
\hline & D443@OD1 & :DSN@H9-:DSN@O4 & 99.95 & 0.2700 \\
\hline & W1@o & :DSN@H19-:DSN@O7 & 99.90 & 0.2707 \\
\hline & D443@OD2 & :DSN@H7-:DSN@O3 & 99.50 & 0.2754 \\
\hline & D571@OD2 & :W1@H2-:W1@O & 99.25 & 0.2729 \\
\hline & D542@OD2 & :DSN@H13-:DSN@O6 & 97.80 & 0.2716 \\
\hline & DSN@08 & :H600@HE2-:H600@NE2 & 77.85 & 0.3027 \\
\hline & D443@OD2 & :DSN@H25-:DSN@O9 & 72.75 & 0.2797 \\
\hline & D443@OD2 & :DSN@H9-:DSN@O4 & 40.75 & 0.3234 \\
\hline & DSN@07 & :R598@HH22-:R598@NH2 & 36.75 & 0.3259 \\
\hline \multirow[t]{12}{*}{ SK complex } & D327@OD1 & :SK@H2-:SK@O9 & 100.00 & 0.2641 \\
\hline & D327@OD2 & :SK@H7-:SK@O8 & 100.00 & 0.2594 \\
\hline & W2@O & :SK@H4-:SK@O7 & 99.90 & 0.2647 \\
\hline & D443@OD2 & :SK@H5-:SK@O6 & 97.70 & 0.2640 \\
\hline & D203@OD2 & :SK@H6-:SK@O3 & 84.80 & 0.2823 \\
\hline & SK@O6 & :R526@HH12-:R526@NH1 & 73.85 & 0.2940 \\
\hline & SK@08 & :H600@HE2-:H600@NE2 & 52.95 & 0.3102 \\
\hline & D542@OD2 & :W2@H2-:W2@o & 44.30 & 0.2763 \\
\hline & SK@011 & :Y299@HH-:Y299@OH & 26.65 & 0.3006 \\
\hline & SK@010 & :Y299@HH-:Y299@OH & 22.45 & 0.3035 \\
\hline & SK@012 & :Y299@HH-:Y299@OH & 16.35 & 0.3026 \\
\hline & W539@NE1 & :W2@H1-:W2@O & 11.70 & 0.3280 \\
\hline \multirow[t]{4}{*}{ SN complex } & D327@OD2 & :SN@H16-:SN@O8 & 100.00 & 0.2597 \\
\hline & D327@OD1 & :SN@H14-:SN@O9 & 99.65 & 0.2668 \\
\hline & Y299@OH & :SN@H22-:SN@O4 & 98.00 & 0.2924 \\
\hline & SN@O8 & :H600@HE2-:H600@NE2 & 95.40 & 0.2938 \\
\hline
\end{tabular}

The order numbers of the oxygen atoms are shown in Fig.6.

tion between DSN and F575 (Fig.5(B)).

The overturning of $\mathrm{C}^{\prime} \mathrm{OH}$ disrupts the close contacts of DSN with D542 or R526 in the ntMGAM active site and weakens the hydrogen bond interaction between DSN and R526. On the contrary, both SK and DSK have stable interaction with R526. The difference of the activity between DSK and DSN is smaller than that between SK and SN because there is only one factor of the nitrogen substitution to affect the binding affinity of DSN compared with DSK. However, the accumulative effects caused by the nitrogen substitution and the sulfate anion group can change the binding conformation of the inhibitor significantly and impair the interactions between glucosidase and inhibitor substantially.

In order to investigate the interaction between the ring ammonium ion (sulfonium ion) and D443, the distance between D443 and the ring ammonium ion (sulfonium ion) of each inhibitor was examined. We found that there is a stable salt bridge between the ring ammonium ion (sulfonium ion) and D443, which stabilizes the inhibitors, especially DSK and SK. Fig.7 shows that the distance between D443 and the ring sulfonium ion of DSK is about $0.42 \mathrm{~nm}$, which is quite similar to that between D443 and the sulfonium ion of SK. For the DSN and SN complexes, the distances are about 0.5 and $0.6 \mathrm{~nm}$, respectively. The distance for SN reaches equilibrium after 3000 ps and that for DSN after 5000 ps. It suggests that there is strong electrostatic interaction between the sulfonium ion (ammonium ion) and the catalytic nucleophile D443. Therefore, the positively charged centers of the ligands obviously play an important role in ligand binding.

\subsubsection{Role of water in the active site}

Finally, we studied the role of the waters in the active site. The importance of the water molecules in the binding site lies in their ability to mediate the interactions between ligand and protein and form hydrogen-bonding networks that can stabilize 


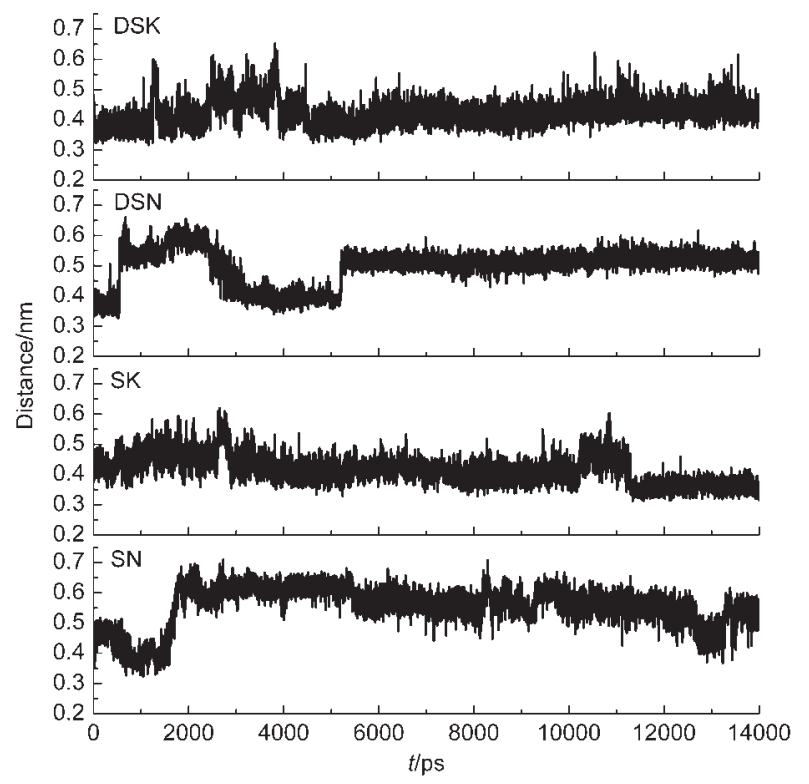

Fig.7 Distance distributions between the CG atom of D443 and the ring $\mathrm{S} 1$ atom of DSK/SK or the $\mathrm{N} 1$ atom of DSN/SN

a protein-ligand complex in solution. Such a network of water molecules may stabilize the complex by forming hydrogen bond with one ligand but not another, and may contribute to the specificity of ligand recognition ${ }^{49}$ and help to stabilize the conformation of the active sites of proteins. ${ }^{50}$ Some water molecules are observed in the active site of the ntMGAM-inhibitor crystal structures. According to our MD simulations, these crystal water molecules can be roughly classified into two categories: the water molecules in category 1 are generally conserved in the active site and can form stable hydrogen bonds with inhibitor and residues, and the water molecules in category 2 are not conserved and have high mobility. Fig.5(A) shows that there are two water molecules (W1 and W2) to mediate the interactions between DSK and protein by forming hydrogen bonds: W1 connects DSK and the residues D571, N543, and W2 connects DSK and two residues S297, H600. In the SK complex W2 moves from W406 to D571 after the MD simulations. In the DSN complex there is also a conserved water molecule that connects DSN and two residues D571, D443. The analysis of the distributions of the water molecules in the active site shows that at least one important water molecule can be found to connect protein and inhibitor by hydrogen bonds in the DSK complex (W1 and W2), the DSN complex (W1), and the SK complex (W2). However, in the binding site of the SN complex we cannot find the conserved water molecule. Undoubtedly, these conserved molecules are important to stabilize the inhibitors in the active site of protein.

The change of the distance between the conserved water molecules and the $\mathrm{O} 7$ atom of inhibitor is shown in Fig.8. We observe that the distance between the conserved water molecules and the $\mathrm{O} 7$ atom of inhibitors keeps stable at $0.25-0.35$ $\mathrm{nm}$ from 7.5 to $14 \mathrm{~ns}$ in the DSK, DSN, and SK complexes, indicating that hydrogen bonding interaction between them is
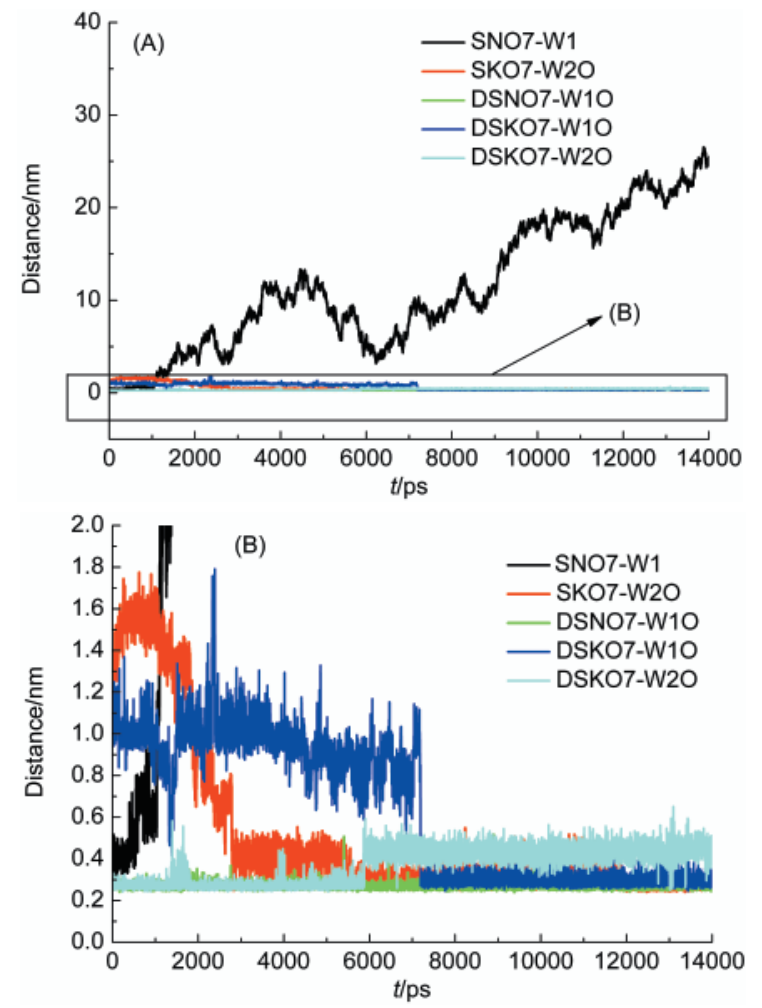

Fig.8 Distance distributions between the 07 atom of inhibitors and the $O$ atom of the conserved water molecules in

$14 \mathrm{~ns}$ MD simulations when the maximum distance along $y$ axis is (A) 400 or (B) 20

O7: the $\mathrm{O} 7$ atom in inhibitors; W1, W20: the $\mathrm{O}$ atom in water $\mathrm{W} 1$ or $\mathrm{W} 2$

stronger than that in the SN complex. This result is in agreement with the content of Table 3.

Therefore we think that the conserved water molecules are important for the binding of this class of inhibitors. They can bridge the interactions between inhibitor and protein by forming a hydrogen bonding network.

\section{Conclusions}

In the current work, a molecular modeling study by combining MD simulations, MM/GBSA binding free energy calculations, and free energy decomposition analysis was employed to investigate the binding mechanisms of four representative inhibitors of glucoamylase. The dynamic binding process of the inhibitors was well characterized by the MD simulations. On the basis of the MD simulations, the binding free energies were predicted using MM/GBSA and the predicted binding free energies are consistent with the experimental values. Our results show that the differences of the polar contribution $\left(\Delta E_{\text {ele }}+\right.$ $\Delta G_{\mathrm{GB}}$ ) between the four complexes may lead to the difference of their inhibitory activity. The free energy decomposition has provided the critical and favorable interaction between glucosidase and the inhibitors. Our simulations highlight the significant impact caused by the combination of the nitrogen substitution and the sulfate anion group. The nitrogen substitution leads to the overturning of the polyhydroxylated chain originat- 
ed from the shorter bond length of $\mathrm{N}-\mathrm{C}$ than $\mathrm{S}-\mathrm{C}$, and the sulfate anion group restrains the freedom of the polyhydroxylated chain. The accumulative effects caused by the nitrogen substitution and the sulfate anion group can change the binding conformation of the inhibitor significantly and impair the interactions between glucosidase and inhibitor substantially. The structural insights obtained from this study are expected to be valuable for the understanding of the binding mechanism of sulfonium ion glucosidase inhibitors and the design of more potent inhibitors of glucosidase.

\section{References}

(1) Nichols, B. L.; Avery, S.; Sen, P.; Swallow, D. M.; Hahn, D.; Sterchi, E. Proc. Natl. Acad. Sci. U. S. A. 2003, 100, 1432. doi: 10.1073/pnas.0237170100

(2) Auricchio, S.; Semenza, G.; Rubino, A. Biochim. Biophys. Acta 1965, 96, 498.

(3) Semenza, G.; Auricchio, S.; Rubino, A. Biochim. Biophys. Acta 1965, 96, 487.

(4) Holman, R. R.; Cull, C. A.; Turner, R. C. Diabetes Care 1999, 22, 960. doi: $10.2337 /$ diacare.22.6.960

(5) Silva, C. H.; Taft, C. A. J. Biomol. Struct. Dyn. 2004, 22, 59. doi: 10.1080/07391102.2004.10506981

(6) Withers, S. G.; Namchuk, M.; Mosi, R. Potent Glycoside Inhibitors: Transition State Mimics or Simply Fortuitous Binders? In Iminosugars as Glycosidase Inhibitors; Arnold, E. S. Ed.; Wiley: New York, 2004; p 188.

(7) Matsuda, H.; Murakami, T.; Yashiro, K.; Yamahara, J.; Yoshikawa, M. Chem. Pharm. Bull. 1999, 47, 1725. doi: 10.1248/cpb.47.1725

(8) Wang, P. Y.; Kaneko, T.; Wang, Y.; Sato, A. Hepatology 1999, 29, 161. doi: 10.1002/hep.510290109

(9) Yoshikawa, M.; Xu, F. M.; Nakamura, S.; Wang, T.; Matsuda, H.; Tanabe, G.; Muraoka, O. Heterocycles 2008, 75, 1397. doi: 10.3987/COM-07-11315

(10) Yoshikawa, M.; Murakami, T.; Shimada, H.; Matsuda, H.; Yamahara, J.; Tanabe, G.; Muraoka, O. Tetrahedron Lett. 1997, 38, 8367. doi: 10.1016/S0040-4039(97)10270-2

(11) Matsuda, H.; Li, Y.; Murakami, T.; Matsumura, N.; Yamahara, J.; Yoshikawa, M. Chem. Pharm. Bull. 1998, 46, 1399. doi: 10.1248/cpb.46.1399

(12) Muraoka, O.; Xie, W. J.; Tanabe, G.; Amer, M. F. A.; Minematsu, T.; Yoshikawa, M. Tetrahedron Lett. 2008, 49, 7315. doi: $10.1016 /$ j.tetlet.2008.10.036

(13) Minami, Y.; Kurlyarna, C.; Ikeda, K.; Kato, A.; Takebayashi, K.; Adachi, I.; Fleet, G. W. J.; Kettawan, A.; Karnoto, T.; Asano, N. Bioorg. Med. Chem. 2008, 16, 2734. doi: 10.1016/j. bmc.2008.01.032

(14) Mohan, S.; Pinto, B. M. Carbohydr. Res. 2007, 342, 1551. doi: 10.1016/j.carres.2007.05.014

(15) Sim, L.; Jayakanthan, K.; Mohan, S.; Nasi, R.; Johnston, B. D.;
Pinto, B. M.; Rose, D. R. Biochemistry-Us 2010, 49, 443. doi: 10.1021/bi9016457

(16) Mohan, S.; Jayakanthan, K.; Nasi, R.; Kuntz, D. A.; Rose, D. R.; Pinto, B. M. Org. Lett. 2010, 12, 1088. doi: 10.1021/ ol100080m

(17) Yuasa, H.; Izumi, M.; Hashimoto, H. Curr. Top. Med. Chem. 2009, 9, 76. doi: 10.2174/156802609787354270

(18) Case, D.; Darden, T. A.; Cheatham, T. E.; Simmerling, C.; Wang, J.; Duke, R.; Luo, R.; Crowley, M.; Walker, R.; Zhang, W.; Merz, K. M.; Wang, B.; Hayik, S.; Roitberg, A.; Seabra, G.; Kolossváry, I.; Wong, K. F.; Paesani, F.; Vanicek, J.; Wu, X.; Brozell, S.; Steinbrecher, T.; Gohlke, H.; Yang, L.; Tan, C.; Mongan, J.; Hornak, V.; Cui, G.; Mathews, D. H.; Seetin, M. G.; Sagui, C.; Babin, V.; Kollman, P. Amber 11; University of California: San Francisco, 2010.

(19) Frisch, M. J.; Trucks, G. W.; Schlegel, H. B.; et al. Gaussian 03, Revision B.03; Gaussian Inc.: Wallingford, CT, 2004.

(20) Bayly, C. I.; Cieplak, P.; Cornell, W. D.; Kollman, P. A. J. Phys. Chem. 1993, 97, 10269. doi: 10.1021/j100142a004

(21) Wang, J. M.; Wolf, R. M.; Caldwell, J. W.; Kollman, P. A.; Case, D. A. J. Comput. Chem. 2004, 25, 1157. doi: 10.1002/jcc.20035

(22) Duan, Y.; Wu, C.; Chowdhury, S.; Lee, M. C.; Xiong, G. M.; Zhang, W.; Yang, R.; Cieplak, P.; Luo, R.; Lee, T.; Caldwell, J.; Wang, J. M.; Kollman, P. J. Comput. Chem. 2003, 24, 1999. doi: $10.1002 /$ jcc. 10349

(23) Jorgensen, W. L.; Chandrasekhar, J.; Madura, J. D.; Impey, R. W.; Klein, M. L. J. Chem. Phys. 1983, 79, 926. doi: 10.1063/ 1.445869

(24) Darden, T.; York, D.; Pedersen, L. J. Chem. Phys. 1993, 98 , 10089. doi: $10.1063 / 1.464397$

(25) Eslami, H.; Mojahedi, F.; Moghadasi, J. J. Chem. Phys. 2010, 133, 084105. doi: 10.1063/1.3474951

(26) Ryckaert, J. P.; Ciccotti, G.; Berendsen, H. J. C. J. Comput. Phys. 1977, 23, 327. doi: 10.1016/0021-9991(77)90098-5

(27) Wang, J.; Hou, T.; Xu, X. Curr. Comput. Aided. Drug. Des. 2006, 2, 287. doi: 10.2174/157340906778226454

(28) Kuhn, B.; Kollman, P. A. J. Med. Chem. 2000, 43, 3786. doi: 10.1021/jm000241h

(29) Huo, S.; Wang, J.; Cieplak, P.; Kollman, P. A.; Kuntz, I. D. J. Med. Chem. 2002, 45, 1412. doi: 10.1021/jm010338j

(30) Hou, T.; Yu, R. J. Med. Chem. 2007, 50, 1177. doi: 10.1021/ jm0609162

(31) Kuhn, B.; Gerber, P.; Schulz-Gasch, T.; Stahl, M. J. Med. Chem 2005, 48, 4040. doi: 10.1021/jm049081q

(32) Hou, T.; Wang, J.; Li, Y.; Wang, W. J. Comput. Chem. 2011, 32, 866. doi: $10.1002 / j c c .21666$

(33) Hou, T. J.; Xu, Z.; Zhang, W.; McLaughlin, W. A.; Case, D. A.; Xu, Y.; Wang, W. Mol. Cell. Proteomics 2009, 8, 639. doi: 10.1074/mcp.M800450-MCP200

(34) Hou, T. J.; Zhang, W.; Case, D. A.; Wang, W. J. Mol. Biol. 2008, 376, 1201. doi: 10.1016/j.jmb.2007.12.054 
(35) Hou, T. J.; Zhang, W.; Xu, X. J. J. Phys. Chem. B 2001, 105, 5304. doi: 10.1021/jp0044476

(36) Hou, T. J.; Zhu, L. L.; Chen, L. R.; Xu, X. J. J. Chem. Inf. Comp. Sci. 2003, 43, 273. doi: 10.1021/ci025552a

(37) Wang, J. M.; Morin, P.; Wang, W.; Kollman, P. A. J. Am. Chem. Soc. 2001, 123, 5221. doi: 10.1021/ja003834q

(38) Hou, T.; Wang, J.; Li, Y.; Wang, W. J. Chem. Inf. Model. 2011, 51, 69. doi: 10.1021/ci100275a

(39) Still, W. C.; Tempczyk, A.; Hawley, R. C.; Hendrickson, T. J. Am. Chem. Soc. 1990, 112, 6127. doi: 10.1021/ja00172a038

(40) Zhang, W.; Hou, T. J.; Qiao, X. B.; Xu, X. J. Acta Phys. -Chim. Sin. 2003, 19, 289. [章 威, 侯廷军, 乔学斌, 徐䈶杰. 物理化 学学报, 2003, 19, 289.] doi: 10.3866/PKU.WHXB20030401

(41) Weiser, J.; Shenkin, P. S.; Still, W. C. J. Comput. Chem. 1999, 20, 217. doi: 10.1002/(SICI)1096-987X(19990130)20:2<217:: AID-JCC4>3.0.CO;2-A

(42) Onufriev, A.; Bashford, D.; Case, D. A. Proteins 2004, 55, 383 doi: $10.1002 /$ prot.20033
(43) Gohlke, H.; Kiel, C.; Case, D. A. J. Mol. Biol. 2003, 330, 891. doi: 10.1016/S0022-2836(03)00610-7

(44) Sim, L.; Quezada-Calvillo, R.; Sterchi, E. E.; Nichols, B. L.; Rose, D. R. J. Mol. Biol. 2008, 375, 782. doi: 10.1016/j. jmb.2007.10.069

(45) Hou, T.; Qiao, X.; Zhang, W.; Xu, X. J. Phys. Chem. B 2002, 106, 11295. doi: 10.1021/jp025595u

(46) Brady, G. P.; Sharp, K. A. J. Mol. Biol. 1995, 254, 77. doi: 10.1006/jmbi.1995.0600

(47) Yuasa, H.; Saotome, C.; Kanie, O. Trends Glycosci. Glycotechnol. 2002, 14, 231.

(48) Eskandari, R.; Jones, K.; Rose, D. R.; Pinto, B. M. Chem. Commun. 2011, 47, 9134. doi: 10.1039/c1cc13052h

(49) Rejto, P. A.; Verkhivker, G. M. Proteins 1997, 28, 313. doi: 10.1002/(SICI)1097-0134(199707)28:3<313::AID-PROT2>3.0. $\mathrm{CO} ; 2-\mathrm{D}$

(50) Pujadas, G.; Palau, J. Protein Sci. 2001, 10, 1645. doi: 10.1110/ ps. 8201 